\title{
THE DYNAMICS OF THE TROJAN ASTEROIDS
}

\author{
ANDREA MILANI \\ Dipartimento di Matematica, Via Buonarroti 2, I-56127 Pisa, Italia \\ E-mail milani@dm.unipi.it
}

\begin{abstract}
The state of the art in the dynamics of the Trojan asteroids has progressed rapidly, since it has been possible to perform numerical integrations of many orbits for millions of years. Accurate proper elements are now computed by the synthetic method, that is from the output of a numerical integration; their stability, at least for time spans of a few million years, is good. This has allowed identification of Trojan families with an automated procedure closely mimicking the one used in the main belt. Although the families identified in a reliable way are only four, the occurrence of significant collisional evolution, not unlike that of the main belt, can be confirmed. The dynamical structure of the Trojan cloud, including the location of the main secular resonances, can be deduced from the proper elements and frequencies by a simple fit. However, many problems are not solved : the origin of a significant percentage of chaotic orbits showing no indications of instability; the location of the stability boundary of the Trojan cloud; the origin of the high inclination of most Trojans. We also do not know if there are "Trojans" for some other planets beside Jupiter : only one Mars Trojan has been found.
\end{abstract}

We are now aware that the Trojan asteroid cloud is not a small feature of the planetary system, but contains a large enough population to be considered an essential component of the Solar System. Thus the widely different level of knowledge and understanding between the main belt and the Trojan cloud is mostly a consequence of the priorities of our research, rather than a reflection of the relevance of the subject. To some extent these priorities are dictated by the observational bias against the discovery and observation of Trojans, which are both farther away and darker. Another reason is that the Trojans are intrinsically a more difficult problem from a dynamical point of view, because their orbits cross the orbit of Jupiter, and close approaches are avoided only because of the $1: 1$ resonance.

Given the striking successes of the last 20 years in the study of the dynamics of the main belt, it is time to ask whether similar results can be obtained for the Trojan cloud, by applying to this problem a suitable amount of research effort. In particular, we would like to know : (1) if it is possible to develop tools equivalent to the proper elements, which have been successfully used for the main belt; (2) if we can detect and study Trojan asteroid families, with the same level of reliability already achieved in the main belt; (3) if we can understand the dynamical structure of the Trojan cloud, including the overall distribution in the phase space with its boundaries and gaps; (4) if we can understand the dynamical stability of the Trojans over time scales comparable to the age of our Solar System, and the interplay between stability, chaos, and the still unknown dissipative processes active during the formation of the Solar System; (5) if there are "Trojans", that is objects in 1 : 1 libration orbits, for some of the other planets besides Jupiter.

This paper reviews the recent progress on all these subjects, and also lists a number of problems which have not yet been solved. For a review of earlier work, see Shoemaker et al. (1989). 


\section{PROPER ELEMENTS FOR TROJANS}

Proper elements are quantities which are almost constant over a very long time span, e.g. millions of years, for each asteroid moving under the gravitational attraction of the Sun and the major planets. To compute proper elements stable over millions of years requires an accurate theory of the motion of the asteroid applicable over the same time span. For the main belt, such theories do exist, based either on an analytical expansion of the solution into series, or on a semianalytical expansion, using an analytical theory obtained with numerical quadratures (for a more detailed discussion of the definition of proper elements in the different regions of the asteroid belt, see Knežević and Milani, this volume).

For the Trojan-type of orbits, purely analytical theories are very difficult, because the perturbing function is singular at the collisions, and most expansion methods normally used in perturbation theory are strongly divergent for a ratio of semimajor axes close to 1 . An analytical theory can be constructed by expanding in powers of small parameters which are related to the distance of the Trojan from the Lagrangian relative equilibria (either $L_{4}$, leading Jupiter by $60^{\circ}$, or $L_{5}$, trailing at the same distance). The trouble is, the distance of the Trojans from the Lagrangian points is by no means small. The (proper) inclination of a Trojan can be as high as $37^{\circ} .5$, the (proper) eccentricity can be 0.22 , the (proper) amplitude of libration in longitude can be $33^{\circ} .3$ : expansions with these quantities as small parameters cannot be a very effective way of computing a theory for the motion of all the Trojans. The most successful analytical theory for the motion of the Trojans was developed by Érdi (1988; and references therein); it is based on the multiple timescale method, and has been pushed to a rather high order in the libration amplitude; however, it only accounts for the perturbations by Jupiter, assumed to be in a fixed elliptic orbit. A semianalytical theory (modelled on the ones by Williams, 1969, and Lemaitre and Morbidelli, 1994) could in principle be computed, but it requires nontrivial modifications to the procedures well established for main belt asteroids. Thus, proper elements for Trojans, of accuracy adequate for the detection of families, were not available until recently.

This unsatisfactory state of affairs was modified when the speed of digital computers become good enough to allow for the numerical integration of a large set of Trojans; Bien and Schubart (1987) computed the orbits of 41 Trojans for a time span of $\simeq 150,000 \mathrm{yr}$ and represented the results in terms of the orbital parameters $D, e_{p}, i_{p}$ which played the rôle of the proper elements in an analytical theory. The geometric interpretation of these parameters will be described below; the essential point is that the output of the numerical integration for each asteroid is post-processed by passing through a sequence of coordinate tranformations and digital filters. The output is a best fitting model, which represents in a fairly accurate way the behaviour of the orbital elements of the asteroid over the integration time span. These numerical techniques, pioneered by Schubart (1982), were later improved upon in the so called "synthetic theories" used in Project LONGSTOP to describe the behaviour of the outer major planets for $10^{8} \mathrm{yr}$ (Carpino et al., 1987).

Recently a modified synthetic theory technique was used by myself (Milani, 1993) to compute proper elements for 174 Trojan asteroids. Apart from technical 


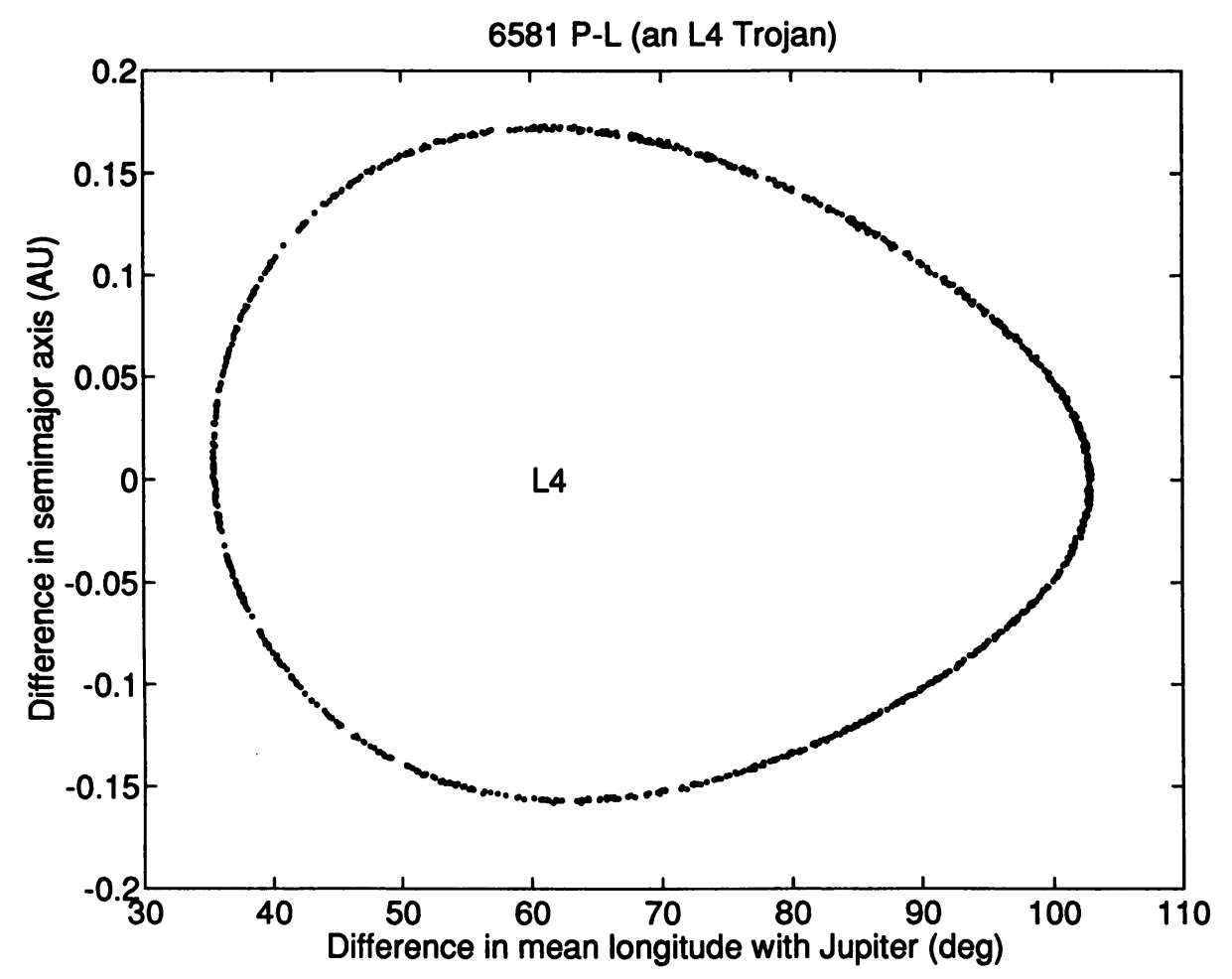

Fig. 1. Libration of the critical argument $\lambda-\lambda^{\prime}$ for the Palomar-Leiden asteroid 6581. The libration amplitude is $0.15 A U$ in semimajor axis, and $33^{\circ} .3$ in mean longitude; these are the largest values found among known Trojans.

improvements (including the use of a $1 M y r$ numerical integration within a realistic model, with the asteroid perturbed by the four giant planets, and the orbits referred to the barycenter of the inner solar System to account for most of the effect of the inner planets), the method is a modification of the one by Bien and Schubart, and the values of the proper elements are consistent for the cases which have been computed also by them, although the definitions are not the same. The main improvement is that I have derived an estimate of the stability in time of the proper elements, by means of the running box method. Proper elements are derived from the first $500,000 \mathrm{yr}$ of output, then by the data from 50,000 to 550,000 yr into the integration, and so on until the last $500,000 \mathrm{yr}$ are used; this provides 11 different estimates, and the maximum excursion (difference between the maximum and minimum) of these 11 values is used as an estimate of the instability in time of the computed proper elements. The results of this stability test, presented in Table 3 of Milani (1993), were already good, with $87 \%$ of the cases with excursions within 0.0025 for $e_{p}, \sin i_{p}$ and within $0.001 A U$ for the proper amplitude of oscillation in semimajor axis $d a$ (corresponding to about $0^{\circ} .2$ for the amplitude of oscillation in longitude): Nevertheless, I have recently recomputed proper elements with an 
1989 U05 (an L5 trojan)

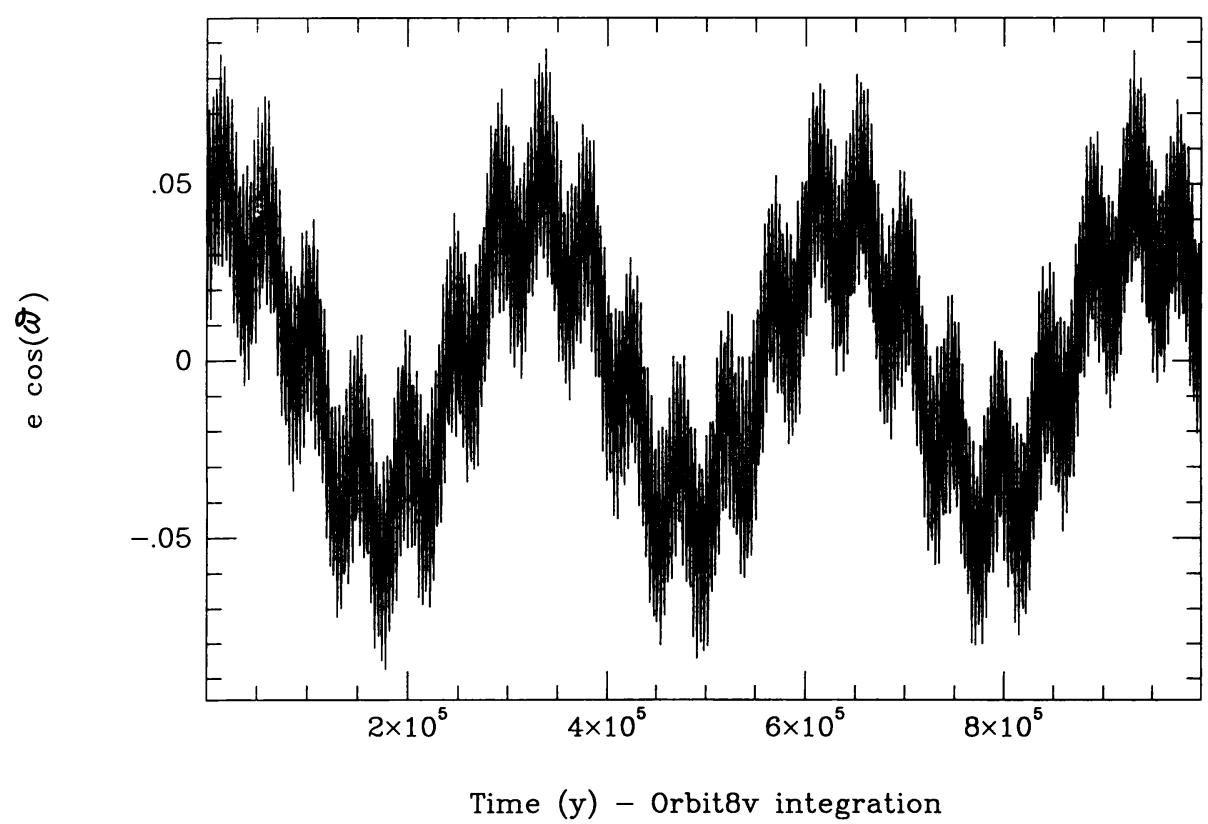

Fig. 2. The variables $h, k$ undergo oscillations with frequencies $g_{5}, g_{6}, \ldots$ (the frequencies of revolution of the perihelia of the major planets), and a proper, or free, oscillation with the much faster proper frequency $g$. The amplitude of the proper mode is, in some cases such as 1989 UO5, less than the amplitude of the forced $g_{5}$ mode: thus the perihelion of the Trojan is locked to the one of Jupiter.

improved algorithm (Version 2.3), which resulted in the same percentage (161 out of 184) of cases with excursions within a reduced range $\left(0.0015\right.$ for $\left.e_{p}, \sin i_{p}\right)$.

The proper elements version 2.3 are defined as follows. The mean elements $a-$ $a^{\prime}, \lambda-\lambda^{\prime}$ (difference with Jupiter in semimajor axis and in mean longitude) are obtained by removing the oscillations with periods up to $50 \mathrm{yr}$ (by means of a digital low-pass filter); their behaviour for a large amplitude libration Trojan is shown in Figure 1. A proper element is the first harmonic component (either da, in the $a-a^{\prime}$ direction, or $D$ in the $\lambda-\lambda^{\prime}$ direction) with respect to the phase angle $\theta$ of the libration. $\theta$ is computed as the polar angle around the Lagrangian point, suitably filtered to remove intermediate period oscillations. This definition of $D$ corresponds to half the value given by Bien and Schubart; the latter is not the difference between maximum and minimum of the critical argument $\lambda-\lambda^{\prime}$, since the libration curve is significantly pear-shaped for large amplitudes (Figure 1).

The proper eccentricity is defined by Fourier analysis of the nonsingular variables $h=e \sin \varpi, k=e \cos \varpi$ (see Figure 2). These variables undergo forced oscillations, 
with frequencies such as $g_{5}, g_{6}$ (periods $\simeq 305,000$ and 45,900 yr respectively) and proper oscillations with frequency $g$ and periods ranging from 3,000 to $5,600 \mathrm{yr}$; given that the frequencies are well separated, it is easy to identify the proper mode by Fourier analysis, and the corresponding amplitude is used as proper eccentricity. To improve the stability of the proper eccentricity, in version 2.3 the Fourier analysis has been performed with respect to the actual value of $\varpi$, rather than with respect to a linear function of time approximating $\varpi$; this is closer to the original definition used by Bien and Schubart.

The proper inclination is defined as the Fourier component with frequency $s$ of the proper oscillations in $p=\sin i \sin \Omega, q=\sin i \cos \Omega$; the main problem is that the frequency $s$ changes over a much wider range that it is the case for $g$ (see Section 3), thus for some Trojans it is necessary to use a very long time series to correctly identify the amplitude of the proper oscillation in $p, q$. To reduce the impact of this problem on the stability of the proper inclination, I have extended the numerical integration to $5 \mathrm{Myr}$ for 20 Trojans.

These results could be improved by carefully analysing the "bad" cases for which the proper elements have a degraded stability, and by introducing a somewhat more complicated synthetic theory to handle most of these. However, the improvements would only concern a few cases; some cases cannot be improved because of the chaotic nature of their orbits (see Section 4). The use of an analytic (or at least semianalytic) theory would also be more informative on the causes of at least some of the less stable cases. However, the brute force work done by means of the numerical integrations such as my own is already good enough to discuss the possible identification of families (Section 2) and the dynamical structure of the cloud, as defined by the lowest degree resonances (Section 3 ). I hope that somebody will be encouraged by the interesting problems raised by this approach and will try to work in a deeper way on the problem of computing a theory for the long-term motion of the Trojans. The proper elements (as well as the results of the running box stability test) are available by anonymous $\mathrm{ftp}$, in the public domain data base described elsewhere in these proceedings.

\section{TROJAN ASTEROID FAMILIES}

Asteroid families are clusterings in the proper elements space, which are too dense to have occurred by chance, and therefore can be interpreted as the result of the catastrophic disruption of a parent body by collision. Since proper elements are stable for millions of years, the disruption might have occurred very long ago, although we do not know when it occurred. For a more complete discussion, see Zappalà and Cellino (these proceedings). The point which is especially relevant for the discussion of possible families among the Trojans, is that the definition of a family requires not only that the asteroids are collisionally interacting, but also that we must be able to find this out by observing and computing now, millions of years after the event. This is an especially demanding requirement, since we know (from the analysis of the families in the main belt, and also from the identification of the parent body of some class of meteorites, as in the case of the eucrites; see Binzel and $\mathrm{Xu}, 1993$; Farinella et al., these proceedings) that the fragments from 
the catastrophic disruption of an asteroid can be ejected with relative velocities of several hundreds of meters per second. In the Trojan cloud it is possible that such fragments could be ejected from the stable libration region and would disappear from the Solar System altoghether, after a close approach to Jupiter. It is therefore clear that the total number of collisionally related groupings is much larger than the number of identifiable families. This difficulty is compounded by the lack of physical observations of Trojans, to the point that we can neither confirm nor discard hypothetical families on the basis of similarities/dissimilarities in the spectra and/or the taxonomic types.

The question I would like to address in this Section is therefore the following : can we find in the data on the orbits of the known Trojans reliable evidence for the existence of families? Does this evidence confirm the occurrence, among the Trojans, of a collisional evolution not unlike the one we know in the main belt? Unlike what was done by Shoemaker et al. (1989), I am here interested in finding proof of the existence of at least some families, rather than proposing many possible families.

The methods to be used for this purpose are well established (Zappalà et al., $1990,1994)$. We need first to choose a metric in the proper elements space; this distance should be related to the impulsive change of velocity needed to change from one orbit to the other. This correspondence is only approximate (see a discussion in Zappalà et al., 1990; Milani et al., 1992). For the case of the Trojan cloud, I have chosen the simplest possible distance, which has the advantage of giving the same weight to the difference in eccentricity and the difference in inclination, without overweighing the difference in semimajor axis, as it is the case for the main metric used by Zappalà et al.. Therefore this metric preserves the peculiar shape of the Trojan "belt" (it is actually a couple of towers, more than $6 A U$ in height) :

$$
d=\sqrt{\frac{1}{4}\left(\frac{\delta d a}{a}\right)^{2}+2(\delta e)^{2}+2(\delta \sin I)^{2}}
$$

The relative velocity corresponding to this distance is simply $V=d \times V_{J u p i t e r} \simeq$ $d \times 13 \mathrm{Km} / \mathrm{s}$; however, this correspondence has to be understood only as an order of magnitude estimate.

The next step is to compute the distance between any two asteroids, and to sort these distances in ascending order. Then we can apply the Hierarchical Clustering Method : we begin by joining all the couples separated by a distance $d$ less than a given value $K$; as $K$ is increased step by step (I have used steps of 0.002 ), a graph is formed, in which the connected components are the candidate groupings. Such a graph is actually shown in Figure 3 (for the "Greeks" around $L_{4}$ ) and Figure 4 (for the "Trojans" around $L_{5}$ ); we have projected on the $e, \sin I$ plane because in the third direction (amplitude of libration in semimajor axis) the Trojan cloud is "thin", and the contribution of the third component to the distances is small.

The procedure needs to be terminated by selecting a maximum value of $K$, otherwise all the points would be clumped together in a meaningless superfamily. The choice of this maximum $K$ must be such that clumps, of the same size as the ones we are interested in, are unlikely to occur by chance in a population of 


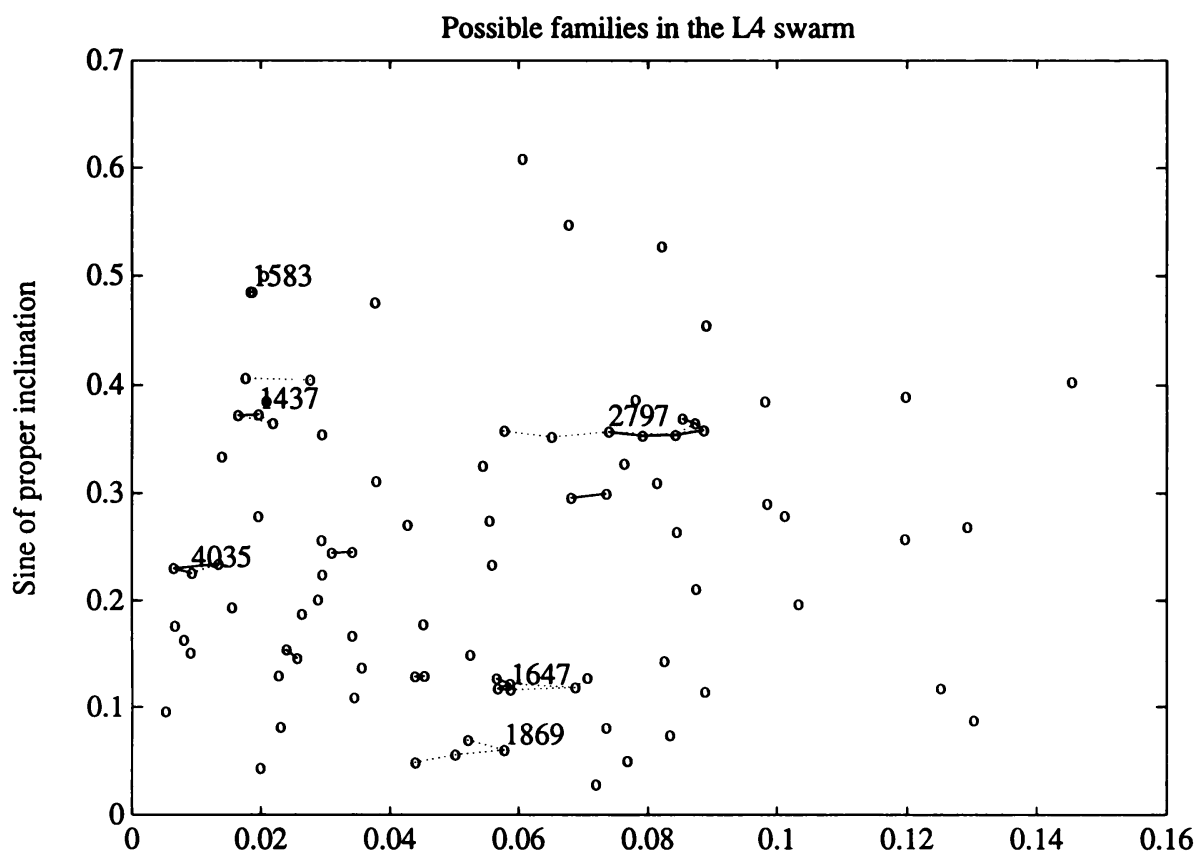

Proper eccentricity (Version 2.3)

Fig. 3. The $L_{4}$ swarm, with a graph indicating the result of the Hierarchical Clustering Method : the solid lines join any two Greeks with a distance $d \leq 0.012$; the groupings of three or more at this distance level should be significant. The dotted lines join couples at a distance $0.012<d \leq 0.016$; these could be collisionally related, especially if they form large groupings and/or connect to already significant families.

objects not collisionally related. To this purpose, Zappalà et al. (1990) introduced the computation of the Quasi Random Level (QRL), which is the value of $K$ at which groupings of a given size would occur by chance in a population with the same overall distribution, but randomized in such a way that there are no real families. The main problem in applying the same method to the Trojans is that the number of orbits in the catalogues is so much smaller than the one for the main belt, that statistical methods based upon random populations might not work, because of statistical fluctuations over a too small sample.

In an attempt to solve this problem, I have introduced a different method to compute the QRL for very small groupings, with only either 2 or 3 members. I have exploited the property of the Trojan population of being subdivided into two independent swarms, around $L_{4}$ and around $L_{5}$, which cannot interact collisionally. Then I can use each of the two swarms as a random clone of the other one; if the distances are computed between all the members of both swarms, then the same 


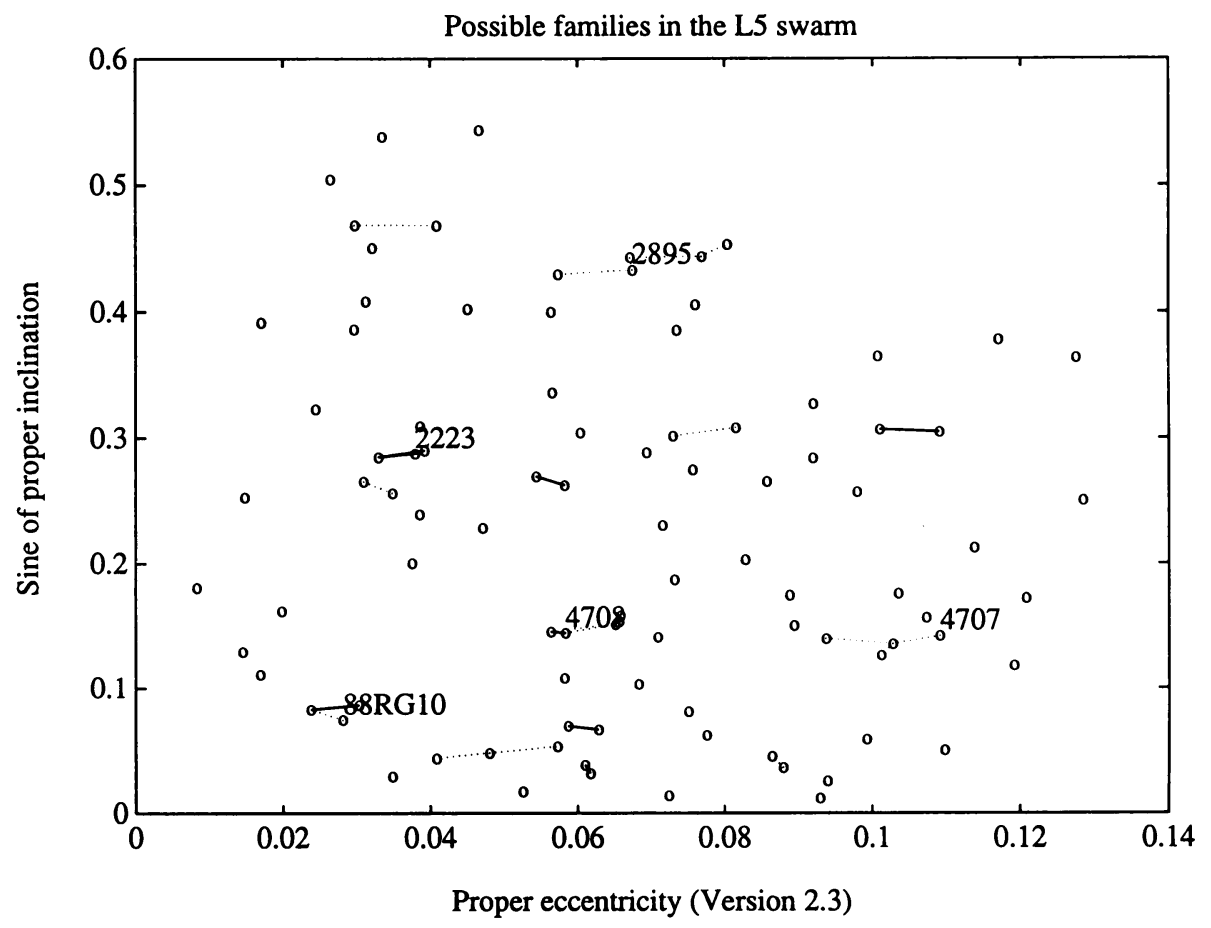

Fig. 4. The $L_{5}$ swarm : in the proper eccentricity/sine of proper inclination plane, the neighbouring couples are joined as in Figure 4. The number of statistically significant clusterings is smaller than around $L_{4}$.

clustering procedure is used for all together, the spurious groupings (with some members in $L_{4}$, some in $L_{5}$ ) play the same rôle of the clusterings found in random samples in the Zappalà et al. method. For the couples, with $K=0.002$ there is only one couple formed by 1583 Antilochus and 3801 Thrasimedes, both in $L_{4}$. However, for $K=0.004$ there are 9 couples, out of which 3 between asteroids in $L_{4}, 6$ spurious (one in $L_{4}$ and one in $L_{5}$ ), and no one in $L_{5}$. The conclusion is that the QRL for couples is less than $\mathbf{0 . 0 0 4}$, and even the couples as close as 0.006 can easily occur by chance, hence cannot be reliable indicators of a collisional event.

The couple $1589-9801$ is very likely to be significant. Actually, the difference in proper elements between 1589 Antilochus and 9801 Thrasimedes is small to the point of raising another problem: it corresponds to $d=0.0006$. When this quantity is translated into relative velocity, it amounts to less than $10 \mathrm{~m} / \mathrm{s}$. Since the escape velocity from 1589 is $\simeq 65 \mathrm{~m} / \mathrm{s}, 3801$ needs to be ejected from 1589 at a speed between 65 and $65.7 \mathrm{~m} / \mathrm{s}$ to escape from the sphere of influence of the larger asteroid, but with such a small relative velocity. Although this is not imposssible, it is unlikely. With P. Farinella I have been studying a non conventional 
collisional model, involving an intermediate stage as binary Trojan, which could explain this couple with a plausible scenario. Such a model has the interesting feature of predicting an observable property of the couple, namely a long rotation period for both asteroids.

A similar analysis of the occurrence of spurious triples shows that for $K=0.012$ there are 8 spurious triples, 1 triple in $L_{5}$ and 8 triples in $L_{4}$. This indicates that the $\mathrm{QRL}$ is $\simeq 0.012$, corresponding to $\simeq 150 \mathrm{~m} / \mathrm{s}$, and also that a significant fraction of the triples in $L_{4}$ does not occur by chance. It follows that the groupings of 3 or more Trojans listed below have to be rated as significant, that is are at least very likely to be families, although it is difficult to quantify the statistical confidence level when working with these small numbers.

In the $L_{4}$ swarm, the Menelaus family includes, besides the numbered 1647 Menelaus, 1973SD1, 1973SQ1, 1989AU1. It is possible that some other asteroids are related to the same family, although the distance is above the QRL : they include the close couple 3548 Eurybates - 6591 PL, and also 1749 Telamon and possibly $91 \mathrm{HN}$ (which has, however, very poor proper elements, both because it has not been observed long enough and because of the $s-s_{6}$ resonance). The Teucer family includes, up to the QRL, 2797 Teucer, 4086, 4946, 2759, 1988BY1 and $1987 Y U 1$; it could have, at a somewhat larger distance, two more members : 3793, 1989AU2. The triple 4035, 1973SM1, 1986TR6 is marginally significant (a clump in Zappalà et al. terminology) because it has exactly 3 members at the QRL for triples. Other groupings, which have been proposed as families by daring authors (including myself), cannot be confirmed by any statistical argument : e.g. Diomedes, Philoctetes.

The $L_{5}$ swarm is surprisingly less rich in significant groupings. Whether this is a subtle observational selection effect or a real difference, is an open question. The Sarpedon family includes only 3 members : 2223 Sarpedon, 1988RR10, 1989SC7; however it is a rather compact triple, with all three mutual distances below 0.012 . The Polydoros grouping consists of two couples, 4708 Polydoros - 4792 Lykaon and 4827 Dares - $4917 P L$, which join one step above the QRL, and therefore does not entirely fulfill the requirement for significance. If they are indeed a family, they might also be related to $4969 T 3$. Other groupings proposed as families, such as Kryses, Memnon and 1988RG10, cannot be confirmed.

Does this procedure answer the main problems, or it is only a tutorial on the use of the Hierachical Clustering Method? The reliable results are only two good families, Menelaus and Teucer, plus the odd couple 1583-3801, in $L_{4}$, and only one reasonably good family, Sarpedon, in $L_{5}$. All these had already been proposed as families (Shoemaker et al., 1989), together with many others which I am unable to confirm with any statistical argument. However, this is what was expected, given the small numbers available (only 94 Trojans and 90 Greeks). An additional limitation is the narrow range in size of the known Trojans : as we know from the main belt, most families have a larger fragment and the others are much smaller. The surveys performed with the large Palomar Schmidt have produced Trojan orbits too inaccurate to be used for family detection, because they used too short obsrvation time spans. On the contrary the survey performed with a small Schmidt by the Shoemakers has given very accurate and useful orbits, but only for compa- 
ratively large asteroids. If we were to look at the main belt at a comparable level of completeness, e.g. if we knew only the orbits of the 603 main belt objects with absolute magnitude $H<10$, we could identify with certainity only the 3-4 major families. The conclusion is that, given the existence of at least four detectable families among the known Trojans, the collisional evolution of the Trojans is not inconsistent with the one of the main belt. To obtain more information, we need to discover/recover a large number of Trojans; when this will be done, the analytical tools will be ready for investigating the collisional evolution of the Trojan swarm.

\section{UNDERSTANDING THE DYNAMICAL STRUCTURE}

The proper elements and the corresponding proper frequencies provide also the right coordinate system in which to look to the distribution of the Trojan orbits. The plots in the plane proper eccentricity/sine of proper inclination (as in Figures 3 and 4) do not show any obvious structure, although it is apparent that most Trojans have proper eccentricities $e_{p}<0.15$; only one object around $L_{4}, 4895$, and one around $L_{5}, 5144$, have $e_{p} \simeq 0.22$ (they are the only ones not shown in the figures because of the smaller window). The inclinations are found in a much wider range. The distribution of the amplitude of libration is more interesting (see Figure 5 ), and the distribution of the frequencies $g$ (of the proper perihelion), $s$ (of the proper node) and $f$ (of the libration phase) clearly indicates the location of some resonances (see Milani, 1993, Figure 11).

The best way to understand the structure of resonances cutting and bounding the Trojan cloud, is to superimpose the asteroid population and the resonance strips computed by some theory providing the frequencies as a function of the proper elements. As an example, this is done in Milani and Knežević (1994), Figures 7-11. However, we do not have a theory to compute the frequencies; we have to resort to a numerical procedure to generate a theory from the experiments (as in Schubart and Bien, 1987). Given the catalogue of proper elements and frequencies for 184 Trojans, I have fit a model in which the frequencies are polynomial expressions in the variables :

$$
x=\frac{e_{p}}{0.15} \quad ; \quad y=\frac{\sin i_{p}}{0.6} \quad ; \quad z=\frac{d a}{0.15 A U}
$$

where the denominators approximate the range of values for each proper element. For the frequency of the proper nodes the best fit gives :

$$
\begin{aligned}
s(\operatorname{arcsec} / \text { year })= & -7.69-7.91 x^{2}+13.36 y^{2}-22.58 z^{2}+ \\
& +2.37 x^{2} y^{2}-1.77 y^{4}-6.33 x^{2} z^{2}+3.96 y^{2} z^{2}-3.51 z^{4}
\end{aligned}
$$

where the $x^{4}$ (that is, $e_{p}^{4}$ ) term has been omitted because it is small and poorly determined; only even powers have been used because they are related to the action variables. The fit for $s$ has an RMS of the residuals of $0.065 \mathrm{arcsec} / \mathrm{yr}$, and is therefore a very good approximation of the behaviour of the frequency as a function of the proper elements. It shows that the frequency $s$ can change significantly, and indeed for the known Trojans $-33.88 \leq s \leq+3.27$, that is, $s$ can actually be zero. The following secular resonances are therefore possible in the Trojan cloud : 


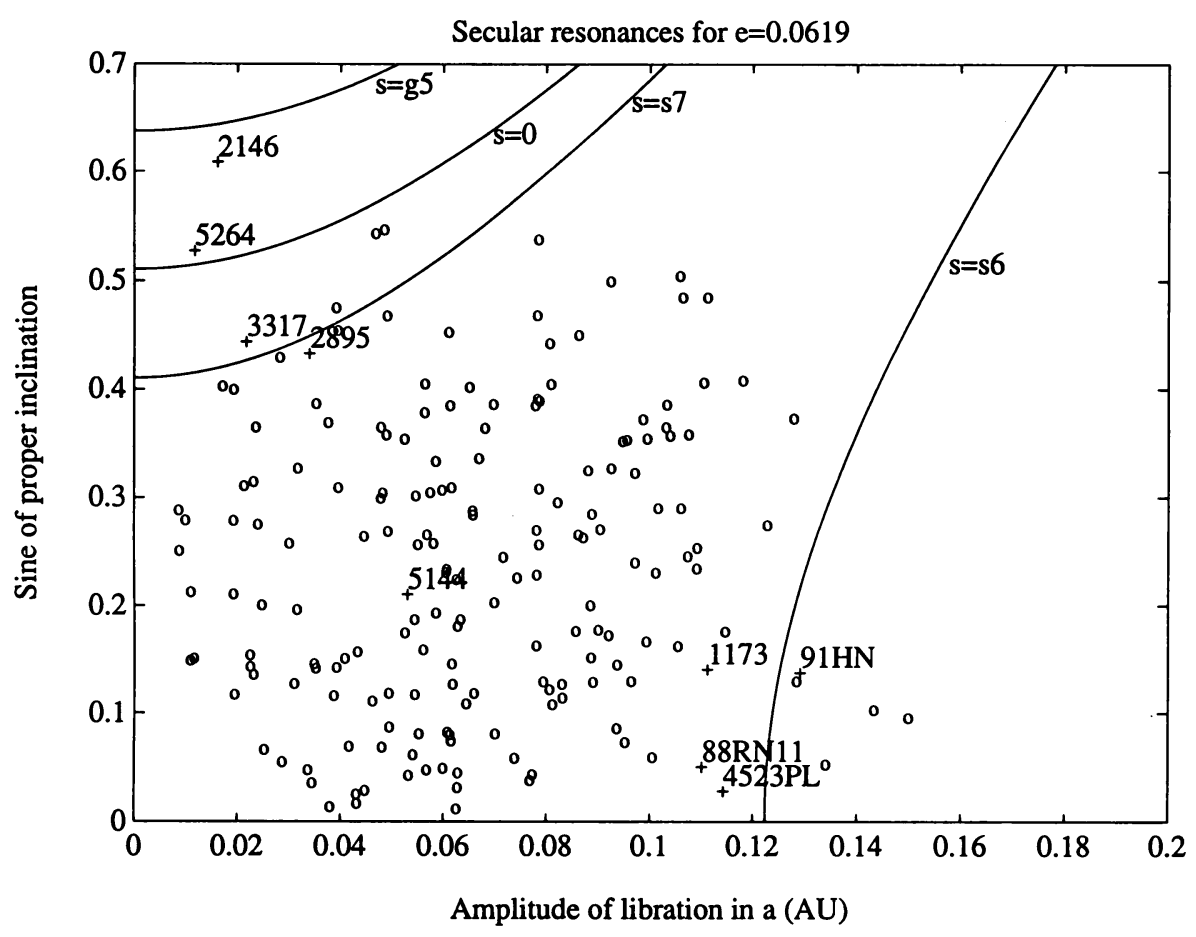

Fig. 5. Secular resonance lines in the plane $d a, \sin i_{p}$, computed for the mean value of $e_{p}$; proper elements for the Trojans with a good stability of $i_{p}$ are plotted as circles. The crosses and the name labels indicate the cases with degraded stability of $i_{p}$.

$s-s_{6}, s-s_{7}$; the resonance $2 s-2 g_{5}$ could also be relevant $\left(s_{i}\right.$ is the proper node frequency, $g_{i}$ the proper perihelion frequency of planet $\left.i\right)$. All these resonances have indeed been proposed to explain the behaviour of some specific asteroid (Bien and Schubart, 1986; Nakai and Kinoshita, 1985). Following the main ideas of these authors, it would be possible to produce a very accurate qualitative model of the behaviour of the orbits in these secular resonances; this problem is especially suitable for the application of semianalytic models.

Figure 5 shows, in the plane sine of proper inclination/amplitude of libration in semimajor axis, the actual Trojans superimposed to the lines corresponding to the main secular resonances involving the node, computed for a value of $e_{p}$ equal to the mean among the real Trojans, that is 0.0619 . The asteroids for which the instability of $\sin i_{p}$ is larger than 0.002 are labelled; they clearly are located near the main resonances ( 5144 also has $s \simeq s_{6}$, but this is not apparent from the Figure, because it has a proper eccentricity very different from the mean value). The Figure also shows that the two main resonances $s-s_{6}, s-s_{7}$ bound the region where most Trojans are. Given the small number of cases, it is not clear whether these secular 
resonances open a gap, or rather define a boundary beyond which there are less asteroids because their dynamical stability is reduced.

Thus the synthetic theory provides us with good clues to understand the dynamical structure, as far as the instability in inclination and the resonances involving the nodes are concerned. The situation is quite different for the eccentricity and the perihelion. A similar fit for the proper perihelion frequency $g$ gives :

$$
\begin{aligned}
g(\operatorname{arcsec} / y r)= & 361.85-12.73 x^{2}-127.81 y^{2}+67.76 z^{2}+ \\
& +2.37 x^{2} y^{2}+5.07 y^{4}-14.17 x^{2} z^{2}-98.09 y^{2} z^{2}-1.85 z^{4}
\end{aligned}
$$

which is also a good fit, with RMS of the residuals of 0.48 arcsec/yr. As it is clear from the dominance of the constant term, $g$ cannot change by a large factor : indeed $231.51 \leq g \leq 423.39 \mathrm{arcsec} / \mathrm{yr}$ for the known Trojans. This implies that the lowest degree resonances involving the proper perihelion of the Trojan cannot occur : as an example, the Kozai separatrix, defined by $2 g=2 s$, beyond which there can be libration of the argument $2 \omega$, cannot occur in the Trojan cloud; also no resonance $g-k g_{i}$ can occur for any planet $i$ and for any integer $k \leq 8$. For the frequency $f$ of the libration phase :

$$
\begin{aligned}
f(\operatorname{arcsec} / y r)= & 8828.8+162.3 x^{2}-1069.8 y^{2}-448.0 z^{2}- \\
& -415.9 x^{2} y^{2}+33.9 y^{4}+105.0 x^{2} z^{2}+21.9 y^{2} z^{2}-269.6 z^{4}
\end{aligned}
$$

with RMS of the residuals of $20.4 \mathrm{arcsec} / \mathrm{yr}$. For known Trojans, $5400 \leq f \leq$ $8896 \mathrm{arcsec} / \mathrm{yr}$, and again no low degree resonance can occur, e.g. $n>k f$ for any $k<12, f>k g$ for any $k<18$. The three proper frequencies $f, g, s$ being very well separated, no low degree resonance between them is possible. This would seem to indicate that the Trojan cloud is little affected by resonances, and most of the phase space should be filled with regular orbits; this fits well with the good stability of the proper elements as shown by the running box test. However, my experience with the problems of very long-term stability of planetary and asteroidal motions made me quite diffident toward this kind of optimistic statements.

\section{OPEN PROBLEMS}

As should be clear from this review, what we know about the dynamics of the Trojan cloud is not very much, although we now have examples to work on from the output of systematic numerical investigations. Rather than giving a long list of questions for which we do not know the answer, I would like to stress three main subjects on which we need some progress in the next few years.

The first problem is the origin of chaos in the orbits of Trojans. During my systematic numerical investigation, I have found 31 cases (out of 184) of positive Lyapounov exponent, with values of Lyapounov time (the time in which two neighbouring orbits diverge by a factor exp(1)) ranging between $15,000 \mathrm{yr}$ and $1 / 6$ of the integration time span. Of course it is not possible to deduce from this that the others are regular orbits, but only that the Lyapounov times, if they exist, 
are longer. The problem is that, out of these 31 chaotic orbits, there is a tentative identifications of the resonance responsible for this behaviour for only a few. 5 cases are near the $s-s_{6}, 3$ near the $s-s_{7}$ resonance; in most of these cases, the proper inclination as computed by the synthetic method is not very stable.

On the contrary, for most cases with a detected positive Lyapounov exponent, I could find no resonance of reasonable degree in which they might be locked. In 5 cases, there was some degradation of the stability of the proper amplitude of libration (between 0.001 and $0.002 A U$ of excursion in the amplitude of oscillation in semimajor axis). In 19 cases, there was no signifcant increase of the instability in any of the three proper elements $d a, e_{p}, \sin i_{p}$, with respect to what was found in the non-chaotic cases (of course, this statement depends upon the accuracy of the technique used to compute the proper elements: with a more accurate method, smaller instabilities might become detectable). In conclusion, more than $10 \%$ of the Trojans exibit some form of stable chaos, that is they behave as if the orbits were constrained to remain close to a quasiperiodic orbit, for a time much longer than the Lyapounov time; this behaviour has also been observed in the main belt (Milani and Nobili, 1992; Milani and Knežević, 1994). These orbits are not accurately predictable, beyond a predictability time horizon which is much smaller than the age of the Solar System; but they are approximately predictable, since the proper elements change within a narrow range over a time much longer than the predictability time.

A theoretical explanation for such a strange behaviour is not yet available, although in principle the stochastic region associated to a chaotic orbit can have any size. The only possible explanation is that these orbits are locked into some resonances of "unreasonable" degree, which result into the full range of chaotic phenomena, including the positive Lyapounov exponent, but has too small a strength to produce a large-scale perturbation (Milani, 1994). The problem is not only to find a mechanism responsible for the chaotic behaviour, but also to assess the possibility of chaotic diffusion in the space of proper elements, over an even longer time scale. This cannot be excluded, since very weak resonances can interact between them, and generate by their combined action an Arnold web along which long-range diffusion is possible. The only sound conclusion is that the stability of these "stable chaos" cases can only be guaranteed for the time span of the numerical tests.

Analytical results on the stability of the orbits of the Trojans are not available; however, interesting results have been obtained with some simplifying assumptions. In the circular restricted three dimensional 3-body model, it has been possible to prove rigorously that the orbits librating around the Lagrangian points with amplitudes up to $\simeq 30,000 \mathrm{Km}$ are stable for a time span longer than the age of the solar system (see Celletti and Giorgilli, 1990, and references therein). Although the results of this kind are not directly applicable to real Trojans with a realistic dynamic model, they indicate that chaotic diffusion can be extremely slow to the point of being astrophysically irrelevant. The point is that this is not necessarily the case; more progress in quantitative estimates of diffusion is necessary.

The second problem is to identify the stability boundaries of the Trojan cloud. It is clear that a Trojan with too large an amplitude of libration could be unstable, because of the possibility of a too close approach to Jupiter; the same is true for 
one with too large an eccentricity. It is also true that chaotic orbits occur more frequently for large libration amplitudes. However, we do not know if there are sharp boundaries, where the Trojan orbits become unstable over timespans much shorter than the age of the solar system, or rather there is a comparatively large transition region from which Trojans can slowly "evaporate" with very long characteristic times, e.g. billions of years. To study the present distribution of the known Trojans is not enough; we need to compute also a number of orbits of hypothetical Trojans, with $e_{p}$ and/or $D$ larger than the observed values.

The main problem is that the structure of these stability boundaries could be very complicated, becoming closer and closer to a fractal as the instabilities time spans increase; thus it is difficult to design an experiment with random initial conditions in such a way that it provides a reliable answer. Computers are now just becoming powerful enough for this kind of "random sample" experiments over millions of years; therefore I will summarize only some recent work, although earlier work should also be quoted (e.g. Innanen and Mikkola, 1989; Mikkola and Innanen, 1992). Holman and Wisdom (1993) have computed the orbits of about 4,000 test particles, initially near the Lagrange points of all the outer planets, for a time span of $20 \mathrm{Myr}$. This was possible also because they used a symplectic mapping method (Wisdom and Holman, 1991) with a stepsize of $1 \mathrm{yr}$; this is of course not an accurate integration, but the advantage, with respect to many previous investigations, is that the approximating dynamical system which is really integrated has the same complexity (e.g., the same number of degrees of freedom, the same possibilities of resonance) of the full problem. They find that the maximum amplitude of libration $d a$ for a low inclination Trojan is about $0.2 A U$, only slightly larger than the maximum value found among the real Trojans. Their experiments also define a large region of stability (for $20 \mathrm{Myr}$ ) for initial conditions around the $L_{4}$ and $L_{5}$ points of Saturn, with the remarkable property that low libration amplitudes $(d a<0.1 \mathrm{AU})$ always result into instability, while intermediate amplitudes $(0.1<d a<0.2 A U)$ can be stable (this was already known to Innanen and Mikkola, 1989). The origin of this instability is not yet known, although I would suggest that a secular resonance could occur for low libration amplitude. Holman and Wisdom also find considerable stability regions around the Lagrange points of Uranus and Neptune; for the latter, they find a puzzling asymmetry between the leading and the trailing side. They conclude, after a discussion of the observational searches conducted so far, that such a "Trojan" of either Saturn, Uranus or Neptune could have been detected, but the degree of completeness of the surveys is low.

Recently, De la Barre and Kaula (1993) have also investigated the stability of ficticious Trojans, both for Jupiter and Saturn, by using an accurate integration of the full outer solar system. They have followed the orbits remaining stable in the Trojan region for up to $161 \mathrm{Myr}$; they find a region of stability which is comparable to the range of libration amplitudes of the real Trojans. Their continuing investigation is now focusing on the high inclination orbits. De la Barre and Kaula also computed about 350 orbits of ficticious Saturn "Trojans", 4 of which remained stable for 329 Myr. They confirm the instability for the Saturn Trojans with low libration amplitude, with timescales for large-scale instability of less than $1 \mathrm{Myr}$; moreover, by computing proper elements (by a synthetic method similar to the one 
I have used), they find that the range of proper eccentricities of the stable Saturn Trojans is very small $\left(e_{p}<0.025\right)$, so that all these orbits have a perihelion locked to that of Saturn and revolving around the Sun with frequency $g_{6}$, not $g$ (as in the example of Figure 2). A tentative conclusion on the existence of Saturn Trojans is that, when all the orbital elements are considered, the volume of the initial conditions in the phase space for stable Saturn Trojans might be quite small, therefore there could be some, but not many of them.

To conclude the discussion on the existence of "Trojans" for other planets, I need to mention that one such object is known for Mars : asteroid 5261 Eureka; it has a rather large inclination $\left(\simeq 20^{\circ}\right)$ and comparatively low eccentricity, with a perihelion longitude close to that of Mars. Mikkola and Innanen (1993) have investigated the range of initial conditions for stable Mars Trojan orbits (for up to $4.4 \mathrm{Myr}$ ) and found that only for inclinations $15^{\circ}<i<30^{\circ}$ and $32^{\circ}<i<44^{\circ}$, and for low to moderate eccentricities, stability is possible. Finally, one Earth Trojan has at least tentatively been identified. Milani et al. (1989) report that, in computing the orbit of the asteroid 2063 Bacchus in the past, they find that it was in the 1 : 1 resonance with the Earth between $-51,000$ and $-10,000$ years before the present time. Since the orbit of Bacchus is highly chaotic in its present state of Geographos-class Earth crosser, any orbital computation for such a long time has only a probabilistic value : we can only conclude that such a state is possible for Bacchus, and might occur for other Near Earth Objects as well.

The third subject on which I would like to see some progress is the understanding of the origin of the orbital distribution of the Trojans. The main puzzle is the high average inclination of the Trojans, and also the extremely high values found for some of them. It is not enough to say that these orbits appear to be stable : we need to know how they can have arisen in the first place. I know of very little work on this subject in the last few years; the main reference is still Yoder (1979), were we find an explanation based upon the sweeping of the $s-s_{6}$ secular resonance. However, the discussion by Yoder should be updated taking into account what we now know on proper elements and on the stability boundary.

\section{Acknowledgements}

I am grateful to P. Farinella, J.G. Williams, D. Davis and C. Froeschlé for carefully reading the manuscript and suggesting many improvements.

\section{References}

Bien, R. and Schubart, J. : 1986, "Two Trojans near a secular resonance : (1173) Anchises and (1871) Astyanax". In Asteroids Comets Meteors II (Lagerkvist C.-I., Rickman, H., Eds.), pp. 157-160, Uppsala University.

Bien, R. and Schubart, J. : 1987, "Three characteristic parameters for the Trojan group of asteroids". Astron. Astrophys. 175, 292-298.

Binzel, R.P. and Xu, S. : 1993, "Chips off of asteroid 4 Vesta : evidence for the parent body of basaltic achondrite meteorites." Science, 260, 186-190.

Celletti, A. and Giorgilli, A. : 1990, "On the stability of the lagrangian points in the spatial restricted problem of three bodies". Celest. Mech. 50, 31-58. 
Carpino, M., Milani, A. and Nobili, A.M. : 1987, "Long-term numerical integrations and synthetic theories for the motion of the outer planets". Astron. Astrophys. 181, 182-194.

De la Barre, C. and Kaula, W. : 1993, "An investigation into the existence of Saturnian Trojan asteroids". Division on Dynamical Astronomy, AAS, 1993 Meeting, Santa Barbara, California.

Érdi, B. : 1988, "Long periodic perturbations of Trojan asteroids". Celest. Mech. 43, 303-308.

Farinella, P., Gonczi, R., Froeschlé, Ch. and Froeschlé, C. : 1993, "The injection of asteroid fragments into resonances". Icarus 101, 174-187.

Holman, M.J. and Wisdom, J. : 1993, "Dynamical stability in the outer solar system and the delivery of short period comets". Astron. J. 105, 1987-1999.

Innanen, K.A., and Mikkola, S. : 1989, "Studies on solar system dynamics. I. The stability of Saturnian Trojans." Astron. J 97 900-908.

Lemaitre, A., and Morbidelli, A. : 1994, "Calculation of proper elements for high inclined asteroidal orbits". Celest. Mech., in press.

Mikkola,S. and Innanen, K.A. : 1992, "A numerical exploration of the evolution of Trojantype asteroidal orbits". Astron. J. 104, 1641-1649.

Mikkola, S. and Innanen, K.A. : 1993, "On the stability of martian trojans". Preprint, Turku University.

Milani, A. : 1993, "The Trojan asteroid belt : proper elements, stability, chaos and families". Celestial Mechanics 57, 59-94.

Milani, A. : 1994, "Proper elements and stable chaos." In : From Newton to chaos : modern techniques for understanding and coping with chaos in $N$-body dynamical systems (A. E. Roy, B.A. Steves, Eds.), Plenum, New York, in press.

Milani, A., and Knežević, Z. : 1994, "Asteroid proper elements and the dynamical structure of the asteroid main belt." Icarus, in press.

Milani, A., and A. M. Nobili : 1992, "An example of stable chaos in the solar system". Nature 357, 569-571.

Milani, A., Carpino, M., Hahn, G. and Nobili, A.M., 1989, Dynamics of planet-crossing asteroids : classes of orbital behaviour, Project SPACEGUARD, Icarus 78, 212-269.

Milani, A., Farinella, P. and Knežević, Z. : 1992, "On the search for asteroid families". In Interelations between physics and dynamics for minor bodies in the solar system, (D. Benest, C. Froschlé, Eds.), 85-132, Editions Frontières.

Nakai, H. and Kinoshita, H. : 1985, "Secular perturbations of asteroids in secular resonance". Celestial Mechanics 36, 391-407.

Shoemaker, E. M., Shoemaker, C. S. and Wolfe, R.F. : 1989, "Trojan asteroids : population, dynamical structure and origin of the L4 and L5 swarms". In Asteroids II (R.P. Binzel, T. Gehrels, M.S. Mattews, Eds.), pp. 487-523, Arizona University Press.

Schubart, J. 1982, "Three characteristic parameters of orbits of Hilda type asteroids". Astron. Astrophys. 114, 200-204.

Schubart, J. and Bien, R. : 1987, "Trojan asteroids : relations between dynamical parameters". Astron. Astrophys. 175, 299-302.

Williams, J.G. : 1969, "Secular perturbations in the Solar System". Ph.D. Thesis, Univ. California Los Angeles.

Wisdom, J. and Holman, M.J. : 1991, "Symplectic maps for the N-Body problem." Astron. J. 102, 1528-1538.

Yoder, C. F. : 1979 " Notes on the origin of the Trojan asteroids." Icarus 40, 341-344.

Zappalà, V., Cellino, A., Farinella, P. and Knežević, Z. : 1990, "Asteroid families I : identification by hierarchical clustering and reliability assessment", Astron. J. 100, 2030-2046.

Zappalà, V., Cellino, A., Farinella, P. and Milani, A. : 1994, "Asteroid families : II. Extension to unnumbered multi-opposition asteroids." Astron. J. 107, 772-801. 